\title{
HERNIACIÓN INTRATORÁCICA TRAUMÁTICA DEL RIÑÓN IZQUIERDO
}

\author{
M. PASCUAL SAMANIEGO, L. FERNÁNDEZ DOMÍNGUEZ, J. CALLEJA ESCUDERO, \\ M.D. RIVERO MARTÍNEZ, F.J. SANZ LUCAS, E. FERNÁNDEZ DEL BUSTO
}

Servicio de Urología. Hospital Clínico Universitario. Valladolid.

Actas Urol Esp. 27 (3): 229-233, 2003

\section{RESUMEN}

"HERNIACIÓN INTRATORÁCICA TRAUMÁTICA DEL RIÑÓN IZQUIERDO"

La aparición de un riñón intratorácico como consecuencia de un traumatismo es un hallazgo excepcional. En la revisión de otros casos publicados en la literatura hay habitualmente un dilatado periodo de latencia hasta la manifestación clínica del proceso, por lo que se planteaba la posibilidad de que el incremento de la presión abdominal fuera únicamente el causante del ascenso hacia el tórax del riñón, a través de un trayecto congénito previamente existente y no causado por el traumatismo. Para descartar esta posibilidad y demostrar el origen traumático de la brecha diafragmática se ha recurrido al examen intraoperatorio de las características de los bordes del orificio herniario.

Así mismo se ha sugerido que el ascenso del riñón sea progresivo en el tiempo, como consecuencia de la presión positiva abdominal y negativa intratorácica.

Nuestro objetivo es presentar un caso extraordinario de traumatismo abdominal en el que la clínica y el estudio radiológico permitieron un diagnóstico inmediato y la constatación quirúrgica del ascenso directo del riñón izquierdo al tórax a través de un orificio herniario diafragmático de nueva adquisición como consecuencia del propio traumatismo.

PALABRAS CLAVE: Riñón intratorácico. Traumatismo. Hernia diafragmática.

\section{ABSTRACT \\ "INTRATHORACIC TRAUMATIC HERNIATION OF THE LEFT KIDNEY"}

The detection of a intrathoracic kidney is an exceptional discovery. There is a large period of time between the injury and its clinical presentation revising the literature, so it is possible that the herniation of the kidney into the chest was caused by a rise in intra-abdominal pressure but going through a preexistent congenital way not caused by the traumatism. In order to dismiss this possibility and to prove the traumatic origin of the diaphragmatic rupture, surgeons have examinated the intraoperative characteristics of the rupture's borders.

The elevation of the kidney has been said to be progressive because of the possitive intra-thoracic pressure and the negative intra-abdominal one.

Our objetive is to report an extraordinary case of abdominal traumatism whose clinic and radiological proofs let us get a rapid diagnosis and surgical demonstration of the direct herniation of the left kidney into the chest through a diaphragmatic rupture caused by the same traumatism.

KEY WORDS: Intrathoracic kidney. Traumatism. Diaphragmatic hernia. 
$\mathrm{L}$ a herniación del riñón hacia el tórax es un hallazgo clínico muy infrecuente que afecta preferentemente al riñón izquierdo puesto que el hígado protege al diafragma derecho contra los cambios bruscos de presión ${ }^{1}$. La mayoría de los casos descritos coinciden en la existencia de un intervalo de tiempo libre de sintomas entre el antecedente traumático y su manifestación clínica o su hallazgo incidental ${ }^{2-4}$. En estos casos es necesario recurrir al examen intraoperatorio de los bordes del orificio herniario, para diferenciar si el traumatismo provocó la herniación por un trayecto congénito preexistente o si el orificio herniario fue adquirido como consecuencia directa del propio traumatismo.

La hiperpresión brusca sobre el abdomen sumado a la presión negativa intratorácica constituyen el mecanismo de ruptura traumática cerrada al diafragma que provoca la herniación visceral intratorácica ${ }^{5}$. Presentamos el caso de un paciente en el que la sintomatología y las sucesivas pruebas radiológicas llevaron al diagnóstico y tratamiento directo de una herniación traumática renal izquierda al tórax de forma inmediata al traumatismo.

\section{CASO CLÍNICO}

Paciente de 22 años de edad, sin antecedentes clínicos de interés, que ingresa de urgencia a causa de un traumatismo toraco-abdominal izquierdo con mecanismo de aplastamiento por chapa de un camión. A la exploración el paciente estaba consciente, pálido, con un hematoma cutáneo torácico en la pared latero-inferior izquierda, dolor a la palpación en el costado e hipocondrio izquierdos y crepitación por la existencia de un enfisema subcutáneo. En la auscultación pulmonar se apreciaba la ausencia de murmullo vesicular en la base del pulmón izquierdo.

Se monitorizaron sus constantes indicando éstas una situación hemodinámicamente estable: tensión arterial 110/75 mm Hg, frecuencia cardíaca 103 latidos/minuto y saturación de oxígeno $100 \%$. Analítica de sangre: creatinina $1,49 \mathrm{mg} / \mathrm{dl}$, $\mathrm{Hb}: 14,4$ gr/dl, Hto: 44,8\% y 19.900 leucocitos. El sedimento urinario presentaba microhematuria (30-50 hematíes/campo), cilindros hialinos y células renales aisladas.
En la radiografía de tórax (Fig. 1), además del enfisema subcutáneo se apreciaban signos de fisura en la $10^{\underline{a}}$ y $11^{\underline{a}}$ costillas izquierdas junto a una elevación y borramiento del hemidiafragma izquierdo, confirmado también por la ecografía donde no se localizaba el riñón izquierdo en su posición anatómica habitual.

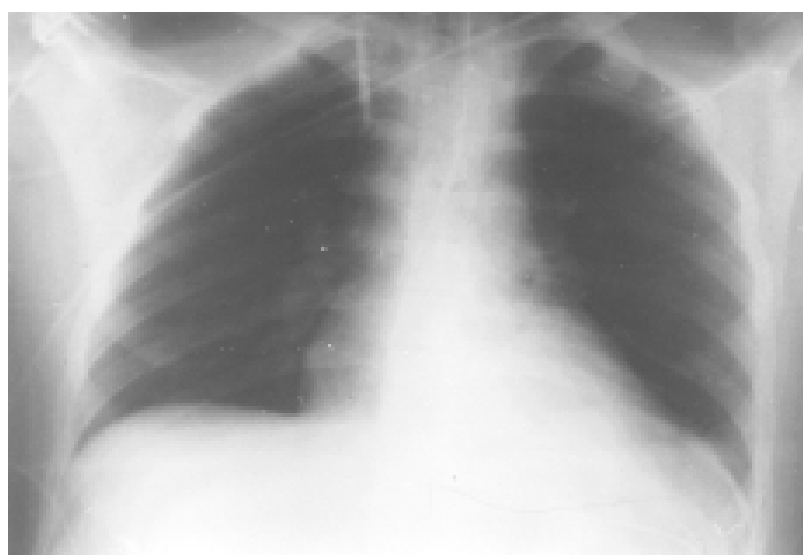

FIGURA 1. Radiografía postero-anterior de tórax. Elevación y borramiento de la cúpula diafragmática izquierda. Se observa parte del enfisema subcutáneo del costado izquierdo.

Dada la situación clínica estable del paciente se procedió a su estudio mediante TC (Tomografía Computerizada) toraco-abdominal. En las imágenes sin contraste intravenoso se demostró la existencia de una masa intratorácica posterior compatible con una herniación traumática al tórax del riñón izquierdo, así como su ausencia en su posición anatómica abdominal (Fig. 2). Tras la inyección de contraste intravenoso se observaba la falta de captación del mismo por el riñón izquierdo intratorácico, manteniéndose la captación y eliminación urinaria por el riñón derecho intraabdominal (Fig. 3).

Ante la sospecha de lesión vascular renal se decidió realizar una arteriografía (Fig. 4), que mostraba una clara imagen de stop en el tercio proximal de la arteria renal izquierda compatible con una isquemia renal trombótica por arrancamiento de la íntima vascular, debido a la elongación traumática arterial.

Tras este estudio se realizó exploración quirúrgica urgente en la que se confirmó la herniación del riñón izquierdo a la cavidad torácica a través de la zona de inserción del pilar posterior del 

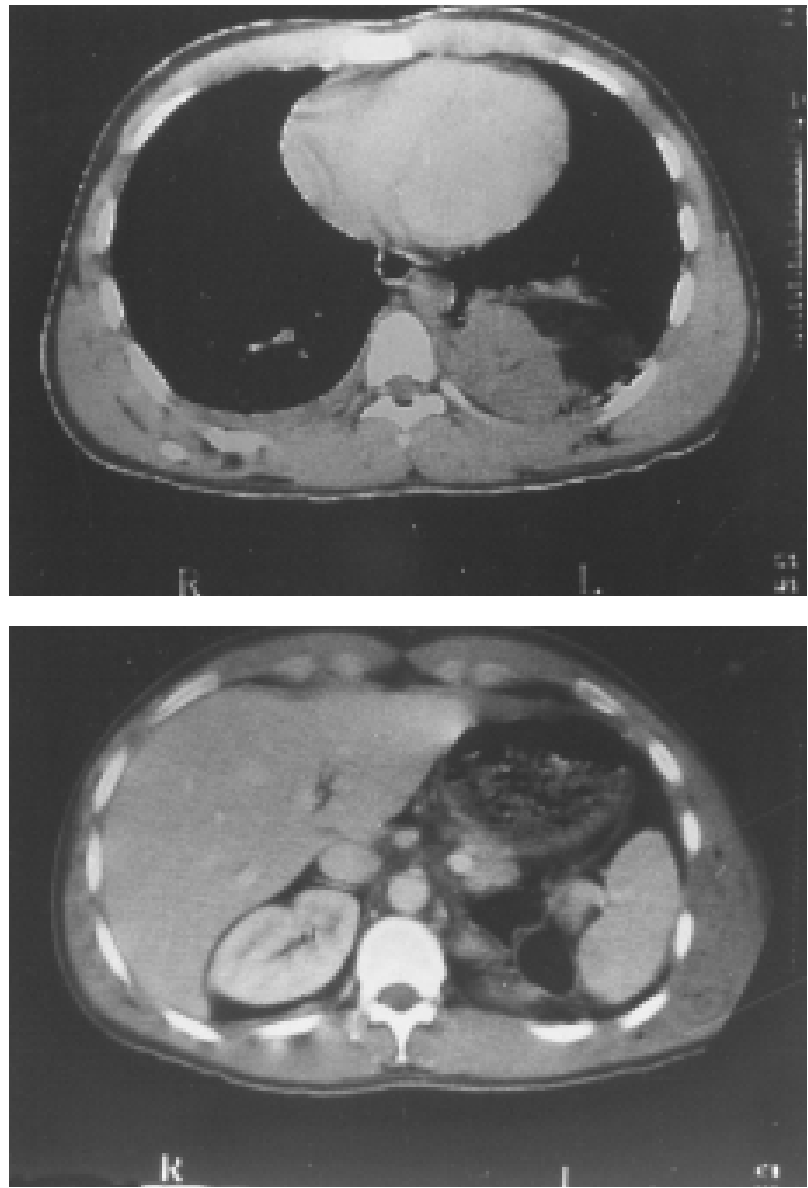

FIGURA 2. Tomografia computerizada. Imagen superior con corte tomográfico a nivel torácico mostrando el riñón izquierdo intratorácico con hematoma acompañante. Imagen inferior con corte tomográfico a nivel abdominal mostrando la ausencia del riñón izquierdo en su posición anatómica habitual.

diafragma, con elongación y verticalización de los vasos renales, constatándose la lesión arterial con ausencia de flujo por lo que se procedió a la nefrectomía izquierda. Además, presentaba hematoma retroperitoneal por arrancamiento de la vena espermática izquierda y rotura múltiple del bazo precisando la esplenectomía. Finalmente se aseguró la recuperación de la integridad diafragmática. La evolución post-quirúrgica fue favorable manteniéndose el paciente asintomático y con buena función renal en las sucesivas revisiones.

El informe de anatomía patológica reflejaba una marcada congestión vascular renal, infiltrado hemático suburotelial de pelvis renal y una gran hemorragia de la pared arterial renal con dislaceración de sus capas y trombo intraluminal.

\section{DISCUSIÓN}

El hallazgo de un riñón intratorácico es una observación muy poco frecuente en la que hay que distinguir inicialmente dos posibilidades en cuanto a su origen. Por un lado la ectopia renal verdadera intratorácica en la que el pedículo vascular renal nace de la aorta descendente intratorácica. Por otra parte, la ascensión renal transdiafragmática al tórax en la que los vasos renales tienen su origen normal en la aorta abdominal, sufriendo una elongación y verticalización como consecuencia del desplazamiento renal ${ }^{3,4}$. En este segundo grupo hay que diferenciar si el orificio herniario es congénito, como posibilidad más frecuente ${ }^{4}$, consecuencia de una aplasia diafragmática afectando
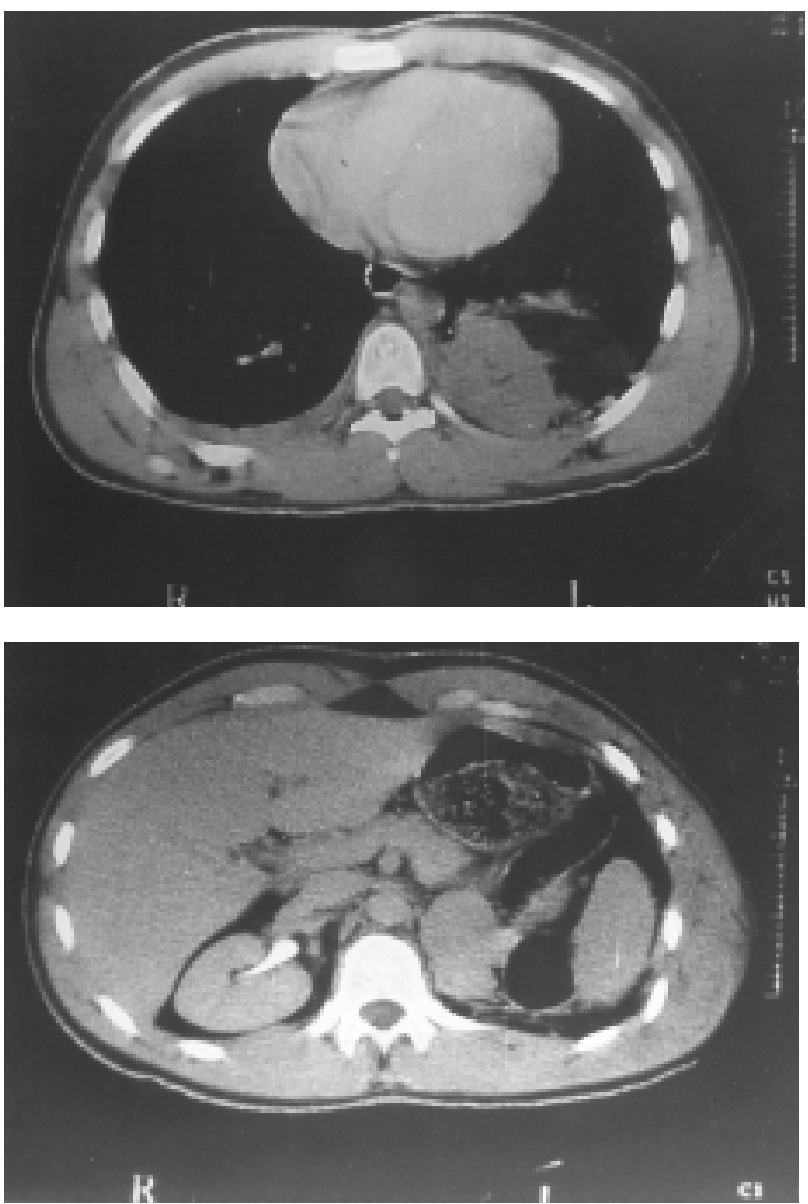

FIGURA 3. Tomografia computerizada con contraste intravenoso. Imagen superior a nivel torácico con ausencia de captación y filtración de contraste por el riñón izquierdo intratorácico. Imagen inferior a nivel abdominal con riñón derecho filtrando el contraste $y$ ausencia intraabdominal del riñón izquierdo. 


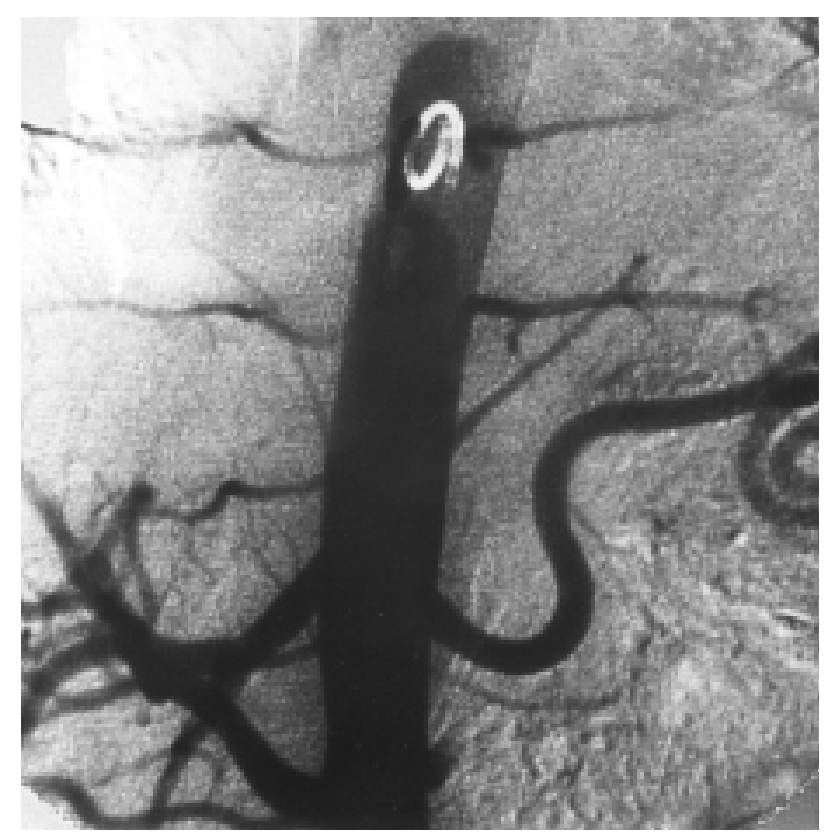

FIGURA 4. Arteriografia de aorta abdominal. Opacificación normal de la arteria renal derecha y ausencia de relleno de la arteria renal izquierda desde su raiz con imagen compatible con trombosis de arteria renal.

principalmente a su porción postero-externa en la zona localizada entre los pilares diafragmáticos, el centro frénico y el reborde $\operatorname{costal}^{2,3}$. Así, la fascia endotorácica tendría continuidad con la de la celda renal estableciéndose una comunicación pleuroperitoneal $^{2,4}$, por la que puede ascender el riñón de una forma progresiva favorecida por la presión negativa intratorácica, o bien por un aumento rápido de la presión intra-abdominal como ocurre en algunos traumatismos ${ }^{5}$.

Pero excepcionalmente, la brecha herniaria puede ser la consecuencia directa de un traumatismo abdominal cerrado como en el caso que presentamos. Estas lesiones son más frecuentes en el hemidiafragma izquierdo debido a la protección hepática contralateral.

La mayoría de los casos descritos coinciden en la existencia de un intervalo libre de tiempo más o menos prolongado entre el antecedente traumático y la presentación de la clínica o el hallazgo incidental del desplazamiento intratorácico renal pudiendo ser bien tolerado por el paciente, lo que sugiere un ascenso renal progresivo debido al agrandamiento escalonado de la brecha inicial diafragmática $^{2}$. Esto coincide con la aparición progresiva de hipertensión arterial (HTA), en cuya fisiopatología se ha implicado a varios mecanismos como la formación de una vaina de esclerosis cicatricial perirrenal, la compresión renal en el orificio herniario y la isquemia por elongación arterial que provoca lesiones glomerulares y tubulares isquémicas identificables por medio de biopsia renal. Dicha HTA puede ser eliminada en aquellos pacientes en los que es factible la reposición del riñón al retroperitoneo ${ }^{3}$.

Sin embargo, hay casos excepcionales como el de nuestro paciente, en los que el incremento brutal de la presión abdominal de forma brusca es el causante directo del orificio diafragmático y del ascenso del riñón en un solo tiempo, produciéndose una elongación por tracción del pedículo vascular y pérdida del riñón por trombosis arterial aguda como mostraba la arteriografía y posteriormente confirmó el estudio anatomopatológico.

Cuando el riñón es funcionante se ha utilizado la urografía intravenosa para realizar diagnóstico diferencial con otras patologías como tumores pulmonares o pleurales primarios o secundarios, y tumores diafragmáticos o de mediastino posterior ${ }^{3}$ habiéndose descrito incluso la provocación de neumotórax para descartar el origen pulmonar de la masa intratorácica ${ }^{2}$. También se ha utilizado la arteriografía para la diferenciación entre ectopia y desplazamiento renal adquirido ${ }^{2,4}$, recurriéndose en este último caso al examen operatorio de los bordes del orificio herniario para distinguir su origen congénito del traumático cuando hay un intervalo libre de tiempo entre el traumatismo y la identificación del riñón intratorácico. Así, en los casos de comunicación congénita los bordes serían lisos, finos y rectos mientras que en el mecanismo traumático son gruesos, irregulares y fibrosos ${ }^{2-4}$. En nuestro paciente no fue necesaria esta diferenciación al no existir duda del origen traumático del orificio herniario por la identificación de forma urgente del riñón intratorácico y de la desinserción parcial del pilar posterior del diafragma.

A pesar del aumento de frecuencia de los traumatismos toraco-abdominales sigue siendo excepcional la presentación de este cuadro patológico. Sin embargo, pensamos que una exploración clínica adecuada junto a la mayor disponibilidad actual para la realización de pruebas radiológicas de urgencia, siempre que la situación clínica lo 
permita, puede llevar a un diagnóstico precoz de la herniación renal intratorácica favoreciendo un tratamiento inmediato que aumente las posibilidades de conservación del riñón. Por otra parte, dichas pruebas ayudan a la valoración relativa de la función renal contralateral cuando es necesaria la nefrectomía por destrucción traumática de la arteria renal, como ocurrió en nuestro paciente.

\section{REFERENCIAS}

1. GRIMES OF.: Traumatic injuries of the diafhragm. Am J Surg 1974; 128: 175.

2. TESTAS P, GOUMET JC.: Hernie thoracique traumatique du rein droit. Ann Urol 1972; 6 (2): 107-111.
3. RIBET M, VOISIN C, LEKIEFFRE J, REMY J, CALLAFE R, TONNEL A.: Hernie diaphragmatique traumatique du rein gauche avec hypertension artérielle. Chirurgie 1974; 100: 237-242.

4. TESTAS P.: Désinsertion traumatique du diaphragme et hernie thoracique du rein droit. Chirurgie 1983; 109: 768-771.

5. LEVY IG, SAADIA R, BOFFARD KD.: Right-sided diaphragmatic rupture with herniation of the kidney prresenting as an uncontrolled haemothorax -a case report. Injury 1988; 19 (4): 293.

Dr. M. Pascual Samaniego

C/ Eusebio González Suárez, 3 - 8으 D 47014 Valladolid

(Trabajo recibido el 15 abril de 2002) 